\title{
High-mass star formation at sub-50 au scales ${ }^{\star}$
}

\author{
H. Beuther ${ }^{1}$, A. Ahmadi ${ }^{1}$, J. C. Mottram ${ }^{1}$, H. Linz ${ }^{1}$, L. T. Maud ${ }^{2,7}$, Th. Henning ${ }^{1}$, R. Kuiper ${ }^{4}, 1$, A. J. Walsh ${ }^{3}$, \\ K. G. Johnston ${ }^{5}$, and S. N. Longmore ${ }^{6}$ \\ ${ }^{1}$ Max Planck Institute for Astronomy, Königstuhl 17, 69117 Heidelberg, Germany \\ e-mail: beuther@mpia.de \\ ${ }^{2}$ Leiden Observatory, Leiden University, PO Box 9513, 2300 RA Leiden, The Netherlands \\ ${ }^{3}$ Research Centre for Astronomy, Astrophysics, and Astrophotonics, Macquarie University, NSW 2109, Australia \\ ${ }^{4}$ Institute of Astronomy and Astrophysics, University of Tübingen, Auf der Morgenstelle 10, 72076 Tübingen, Germany \\ ${ }^{5}$ School of Physics and Astronomy, University of Leeds, Leeds, LS2 9JT, UK \\ ${ }^{6}$ Astrophysics Research Institute, Liverpool John Moores University, 146 Brownlow Hill, Liverpool L3 5RF, UK \\ ${ }^{7}$ European Southern Observatory, Garching bei Munchen, Germany
}

Received 9 August 2018 / Accepted 19 November 2018

\begin{abstract}
Context. The hierarchical process of star formation has so far mostly been studied on scales from thousands of au to parsecs, but the smaller sub-1000 au scales of high-mass star formation are still largely unexplored in the submillimeter regime.

Aims. We aim to resolve the dust and gas emission at the highest spatial resolution to study the physical properties of the densest structures during high-mass star formation.

Methods. We observed the high-mass hot core region G351.77-0.54 with the Atacama Large Millimeter Array with baselines extending out to more than $16 \mathrm{~km}$. This allowed us to dissect the region at sub-50 au spatial scales.

Results. At a spatial resolution of $18 / 40$ au (depending on the distance), we identify twelve sub-structures within the inner few thousand au of the region. The brightness temperatures are high, reaching values greater $1000 \mathrm{~K}$, signposting high optical depth toward the peak positions. Core separations vary between sub-100 au to several 100 and 1000 au. The core separations and approximate masses are largely consistent with thermal Jeans fragmentation of a dense gas core. Due to the high continuum optical depth, most spectral lines are seen in absorption. However, a few exceptional emission lines are found that most likely stem from transitions with excitation conditions above $1000 \mathrm{~K}$. Toward the main continuum source, these emission lines exhibit a velocity gradient across scales of 100-200 au aligned with the molecular outflow and perpendicular to the previously inferred disk orientation. While we cannot exclude that these observational features stem from an inner hot accretion disk, the alignment with the outflow rather suggests that it stems from the inner jet and outflow region. The highest-velocity features are found toward the peak position, and no Hubble-like velocity structure can be identified. Therefore, these data are consistent with steady-state turbulent entrainment of the hot molecular gas via Kelvin-Helmholtz instabilities at the interface between the jet and the outflow.

Conclusions. Resolving this high-mass star-forming region at sub-50 au scales indicates that the hierarchical fragmentation process in the framework of thermal Jeans fragmentation can continue down to the smallest accessible spatial scales. Velocity gradients on these small scales have to be treated cautiously and do not necessarily stem from disks, but may be better explained with outflow emission. Studying these small scales is very powerful, but covering all spatial scales and deriving a global picture from large to small scales are the next steps to investigate.
\end{abstract}

Key words. stars: formation - stars: massive - stars: individual: G351.77-0.54 - stars: winds, outflows instrumentation: interferometers

\section{Introduction}

Star formation is an intrinsically hierarchical process where fragmentation occurs on almost all scales, from the large-scale molecular clouds with sizes of several ten to hundred pc, down to close binary formation at the center of dense cores and disk fragmentation that can lead to planets. In that framework, the smallest scale fragmentation of the cores and potentially also the disks are poorly understood, in particular for regions forming high-mass stars $\left(>8 M_{\odot}\right)$ because they are typically located at distances of several kpc. Until recently, the state-of-the-art observations had as high resolution as $\sim 0.2^{\prime \prime}$, allowing for the investigation of the fragmentation processes of the larger scale

\footnotetext{
* The data are only available at the CDS via anonymous ftp to cdsarc.u-strasbg.fr $(130.79 .128 .5)$ or via http://cdsarc.u-strasbg.fr/viz-bin/qcat?J/A+A/621/A122
}

molecular gas clumps into their cluster-forming cores (e.g., Beuther et al. 2007, 2013, 2018; Bontemps et al. 2010; Palau et al. 2013, 2014; Csengeri et al. 2017; Cesaroni et al. 2017). To understand the formation of close binaries or systems like the Trapezium in Orion (e.g., Preibisch et al. 1999), higher spatial resolution is needed. Furthermore, many other physical processes take place on small spatial scales. For example, disk-like structures as well as the inner jet and outflow regions are found on scales on the order of 10 to a few 100 au (e.g., Tan et al. 2014; Frank et al. 2014; Beltrán \& de Wit 2016), again requiring the highest spatial resolution possible. Additionally important are global infall and streamer-like motions through which the material can get channeled in the innermost regions (e.g., Maud et al. 2017; Goddi et al. 2018).

Millimeter and centimeter wavelengths are the ideal spectral regime to study these processes because the young disks and 

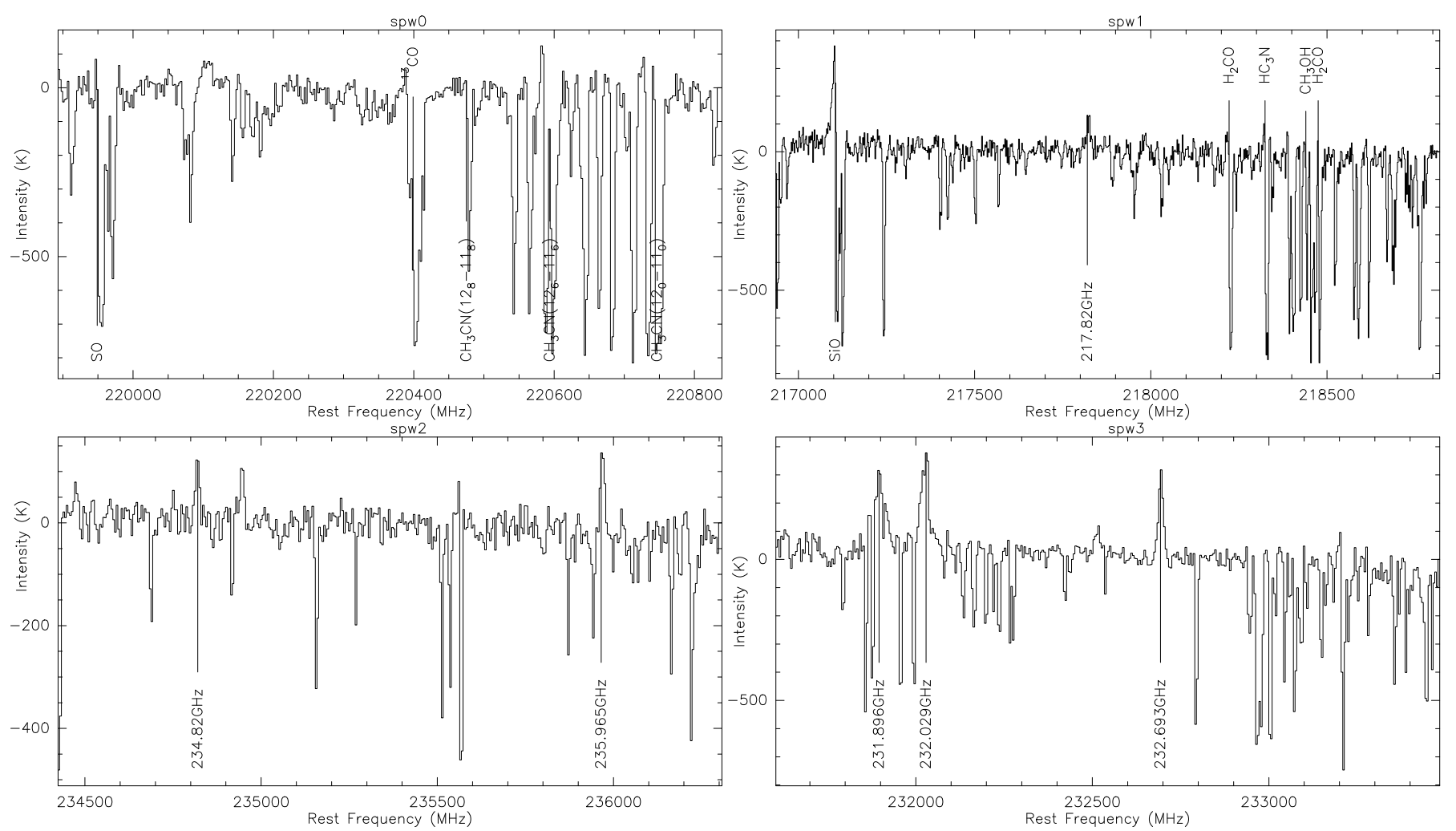

Fig. 1. Spectra of the four spectral windows extracted toward the main mm continuum peak position mm1. Clearly most lines are seen in absorption against the continuum, only a few in emission. Selected spectral lines are labeled.

jets are still deeply embedded within their natal cores. Sub-0.1" spatial resolution can be achieved with the Very Large Array at cm wavelengths (e.g., Moscadelli \& Goddi 2014; Beuther et al. 2017a), and now also with the Atacama Large Millimeter Array (ALMA) at (sub)mm wavelengths (e.g., ALMA Partnership 2015).

Our target region is the well-known high-mass star-forming region G351.77-0.54 (a.k.a. IRAS 17233-3606). The distance to the target is debated, and while early studies put it at $2.2 \mathrm{kpc}$ (Norris et al. 1993), more recent investigations favor smaller distances around $1 \mathrm{kpc}$ (Leurini et al. 2011a). Since the accurate distance is not clear yet, in the following, we will always give the parameters for both distances. The luminosity and mass of the entire region are estimated to be $1.7 \times 10^{4} / 10^{5} \mathrm{~L}_{\odot}$ and $660 / 3170 M_{\odot}$ at $1 / 2.2 \mathrm{kpc}$, respectively (Norris et al. 1993; Leurini et al. 2011a,b). These numbers clearly show that we are dealing with a massive star-forming region on its way to form high-mass stars. The region exhibits linear $\mathrm{CH}_{3} \mathrm{OH}$ class II maser features as well as strong outflow emission (e.g., Norris et al. 1993; Walsh et al. 1998; Leurini et al. 2009, 2013; Klaassen et al. 2015). Furthermore, this region is a very bright (sub)mm line emission source. Centimeter-wavelength interferometric studies revealed strong $\mathrm{NH}_{3}(4,4) /(5,5)$ emission at temperatures above $200 \mathrm{~K}$ and resolving overall rotational motion on a few 1000 au scales (Beuther et al. 2009), approximately perpendicular to the larger scale outflow. Following Leurini et al. (2011a), we use as $v_{\mathrm{lsr}}$ a value of approximately $-3.6 \mathrm{~km} \mathrm{~s}^{-1}$. Since Zapata et al. (2008) found $6 \mathrm{~cm}$ continuum sources within that core, it was expected that this region would show fragmentation on smaller spatial scales in the (sub)mm regime. Furthermore, multiple outflows have been identified (e.g., Leurini et al. 2009, 2011a; Klaassen et al. 2015;
Beuther et al. 2017b). In a recent ALMA study of this region at $690 \mathrm{GHz}$ we resolved the region at $0.06^{\prime \prime}$ resolution (Beuther et al. 2017b). This study revealed at least four sub-cores where the central source \#1 provides strong evidence for rotational motion on scales of a few hundred au, perpendicular to an outflow seen in $\mathrm{CO}(6-5)$. But ALMA can go to even higher spatial resolution, and here we present a study of the same region at $\sim 1.3 \mathrm{~mm}$ wavelengths and 21 mas $\times 15$ mas resolution, corresponding to $\sim 18 / 40$ au at a distance of $1 / 2.2 \mathrm{kpc}$.

\section{Observations}

The target high-mass star-forming region G351.77-0.54 was observed with ALMA as a cycle 3 project (id 2015.1.00496) in four separate observing blocks between Nov. 5 and Dec. 2, 2015. The duration of the four scheduling blocks varied between 1 and $1.6 \mathrm{~h}$ with on-source target observing times between 24 and 45 min. Between 33 and 43 antennas were in the array and baselines between $17 \mathrm{~m}$ and more than $16 \mathrm{~km}$ were covered. The phase center of our observations was RA (J2000.0) 17:26:42.568 and Dec (J2000.0) -36:09:17.60. To calibrate the phases on the long baselines, short cycle times were used between the target and the phase and amplitude calibrator J1733-3722 with a typical cycle times consisting of $\sim 55 \mathrm{~s}$ on source and $\sim 18 \mathrm{~s}$ on the calibration quasar. Bandpass and flux calibration were conducted with the sources J1517-2422 and J1617-5848, respectively. The precipitable water vapor (PWV) varied between 0.42 and $0.93 \mathrm{~mm}$. The $1.3 \mathrm{~mm}$ band 6 receivers were tuned to the LO frequency of $226.378 \mathrm{GHz}$ with two spectral windows (spws) in the upper and lower sidebands, respectively. The frequency coverages of the four spectral windows is depicted in Fig. 1. The spectral resolution of the spws was 244 and $488 \mathrm{kHz}$, and for 
the wider spectral windows $3906 \mathrm{kHz}$, corresponding to velocity resolutions of $\sim 0.33, \sim 0.67$ and $5.3 \mathrm{~km} \mathrm{~s}^{-1}$, respectively. The primary beam of ALMA at these frequencies is $\sim 27^{\prime \prime}$.

The calibration of the data was done with the ALMA pipeline in CASA (McMullin et al. 2007) version 4.5.0 following the scripts provided by the observatory. In the following step, we applied phase-only self-calibration to the data going down to time-steps of $30 \mathrm{~s}$. Images prior and after self-calibration were carefully compared, and while all structures are well recovered, the noise is decreased after self-calibration. No amplitude self-calibration was applied.

The following imaging was also conducted within CASA. Although some baselines between 17 and $55 \mathrm{~m}$ are observed, their coverage is sparsely sampled, and so do not allow us to reasonably image the larger scale emission. Therefore, in the imaging process we only used data from baselines longer than $55 \mathrm{~m}$, which allows us to concentrate on the most compact structures. As visible in Fig. 1, this hot core hosts many spectral lines and defining line-free parts of the spectrum is not easy. Therefore, we compared different approaches to create a continuum image of the region. On the one hand, we produced a continuum image from only a small part of the spectrum that appears line-free, and on the other hand, we produced a continuum image from the whole bandpass with all spectral features included. Structurally and with respect to the intensities, there was barely any difference between the two images, only the noise of the full-bandpass image was lower. Therefore, in the following we use the continuum image created from the whole bandpass. For the continuum images we applied a robust weighting of -2 , corresponding to uniform weighting. This results in a synthesized beam of 21 mas $\times 15$ mas with a position angle of $-74^{\circ}$. A few apparently line-free parts of the spectra were used for the continuum subtraction. However, in such a line-rich source, this is not a trivial process and some weak bandpass slope remains (Fig. 1). However, that slope is so weak that is does not affect any of the analyses. The line data were then imaged with a robust weighting of 0 , resulting in a slightly larger synthesized beam of $\sim 25$ mas $\times 20$ mas with a position angle of $-86^{\circ}$. The continuum $1 \sigma \mathrm{rms}$ is $\sim 50 \mu \mathrm{Jy}_{\text {beam }^{-1}}$, and the typical $\mathrm{rms}$ for the spectral lines in a line-free channel of $1 \mathrm{~km} \mathrm{~s}^{-1}$ width is $\sim 0.9 \mathrm{mJy} \mathrm{beam}^{-1}$.

\section{Results}

As outlined in the previous Sect. 2 and shown in Figs. 1 and 2, extended emission is not well imaged in this long-baseline-only dataset, and most lines are mainly seen in absorption against the continuum sources. However, there are a few emission lines (Fig. 1) showing structure on small spatial scales below $100 \mathrm{au}$. Therefore, in the following we concentrate on the small-scale continuum emission as well as on the few transitions seen in emission.

\subsection{Continuum emission and core fragmentation}

Figure 2 presents a compilation of the $1.3 \mathrm{~mm}$ continuum data of the region. While the central panel shows the inner $\sim 7^{\prime \prime} \times 7^{\prime \prime}$, the surrounding 8 panels present zoom-ins for the sub-structure. With an angular resolution of $0.021^{\prime \prime} \times 0.015^{\prime \prime}$ (mean of $0.018^{\prime \prime}$ ), we can resolve linear structures of roughly $18 / 40$ au at $1 / 2.2 \mathrm{kpc}$ distance, among the highest spatial resolution observations achieved for high-mass star-forming regions today.

While at first sight, this image appears largely empty with only a few compact sub-structures distributed throughout, one should keep in mind that this image is strongly affected by missing flux because we only image data with baselines larger than $55 \mathrm{~m}$ (and extending to more than $16 \mathrm{~km}$ ). Hence, all intermediate and large-scale structures are filtered out and we concentrate on the small sub-cores remaining. To get a feeling for the fraction of missing flux, we extract the $870 \mu$ m flux (central frequency $345 \mathrm{GHz}$ ) of G351.77-0.54 from the ATLASGAL survey (Schuller et al. 2009). Scaling the $870 \mu \mathrm{m}$ peak flux density of $52.1 \mathrm{Jy}^{-1} \mathrm{bam}^{-1}$ (with a beam size of 19.2") with a flux dependency of $S \propto v^{4}$ to our central frequency of $226.6 \mathrm{GHz}$, approximately $9.7 \mathrm{Jy}$ should be emitted in our field. However, as shown below, the sum of the fluxes of all identified subcores accumulates to only $\sim 208 \mathrm{mJy}$. Taking these numbers, we recover only about $2.1 \%$ of the total continuum emission in the field.

Nevertheless, this spatial filtering also has strong advantages because we can isolate the compact structures within this region. With the sparse spatial sampling on short and intermediate baselines, and only a limited amount of emission features in the map, an automized source identification is not required. Therefore, we identify the source structures by eye following the $4 \sigma$ contours in Fig. 2. This way, we identify 12 separate $\mathrm{mm}$ sources within a radius of roughly $3.5^{\prime \prime}$ or $3500 / 7700$ au at $1.0 / 2.2 \mathrm{kpc}$ distance, respectively. Projected separations range from more than 1000 au to several $100 \mathrm{au}$. Some sources, for example, mm1 and $\mathrm{mm} 2$, show even smaller secondary peaks on scales below $100 \mathrm{au}$. The largest extended structures we can trace with these observations are found toward the sub-sources $\mathrm{mm} 1, \mathrm{~mm} 4$ and $\mathrm{mm} 8$, and depending on the distance to the region, these sources have sizes between roughly 200 and $600 \mathrm{au}$. Taking $\mathrm{mm} 9$ as an example, and assuming the closer distance of $1 \mathrm{kpc}$, our spatial resolution corresponds to $\sim 18 \mathrm{au}$, and the extent of the source is roughly $300 \mathrm{au}$. This outlines the approximate spatial dynamic range covered by these data. None of the mm continuum sources are directly spatially associated with the $\mathrm{cm}$ continuum sources reported in Zapata et al. (2008) and marked in Fig. 2. In comparison to the $438 \mu \mathrm{m}$ submillimeter continuum sources presented in Beuther et al. (2017b), the here identified sources $\mathrm{mm} 1$ and $\mathrm{mm} 2$ correspond to their sources \#1 and \#2. The sources \#3 and \#4 in Beuther et al. (2017b) correspond to the mm sources mm12 and $\mathrm{mm} 3$, respectively. While the compact sources most likely host protostars, some of the more diffuse structures like $\mathrm{mm} 5$ or $\mathrm{mm} 7$ may not be of protostellar nature but could rather be remnants of the larger scale envelope where only some imaged structure is left after the interferometric spatial filtering (see also Sect. 4.1).

At these spatial scales, optical depth effects can also become important, and one way to assess this is by converting the peak flux densities into peak brightness temperatures. Table 1 presents for all 11 identified sub-structures the peak flux densities $S_{\text {peak }}$, the integrated fluxes $S_{\text {int }}$ (integrated within the $4 \sigma$ contours), the corresponding peak brightness temperature $T_{\mathrm{b}}$ as well as the mean brightness temperatures derived again within the $4 \sigma$ contours of $16 \mathrm{~K}$. As one sees, the peak brightness temperatures toward the strongest positions $\mathrm{mm} 1$ and $\mathrm{mm} 2$ exceed $1000 \mathrm{~K}$, and toward the other peak positions are between 29 and $310 \mathrm{~K}$. From the $\mathrm{CH}_{3} \mathrm{CN}$ temperature analysis for this region at $690 \mathrm{GHz}$ presented in Beuther et al. (2017b), we know that the gas temperatures around $\mathrm{mm} 1$ are also around $1000 \mathrm{~K}$. This clearly shows that the dust emission in this region is optically thick and traces an inner core surface at the derived brightness temperature. Such high dust optical depth can also be inferred from the fact that almost all lines are seen in absorption against the $\mathrm{mm} 1$ continuum peak (Fig. 1). While the peak brightness temperatures in the other sub-structures are lower, they are still high compared 

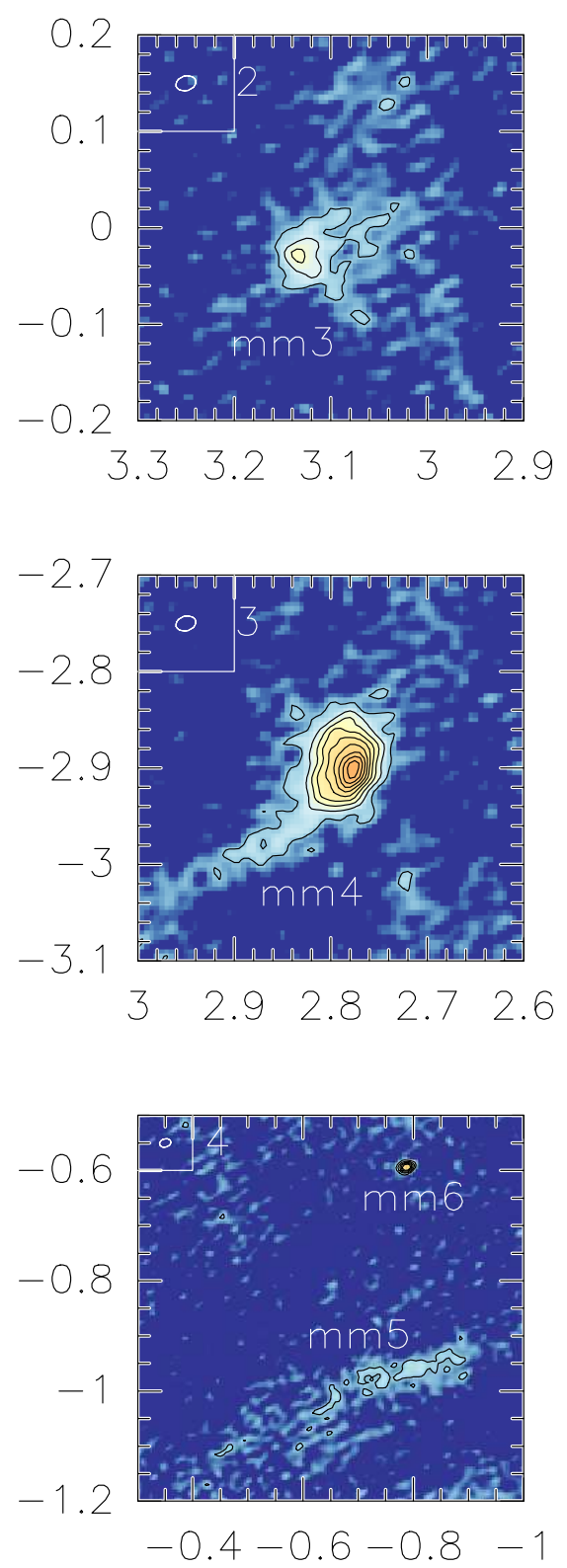
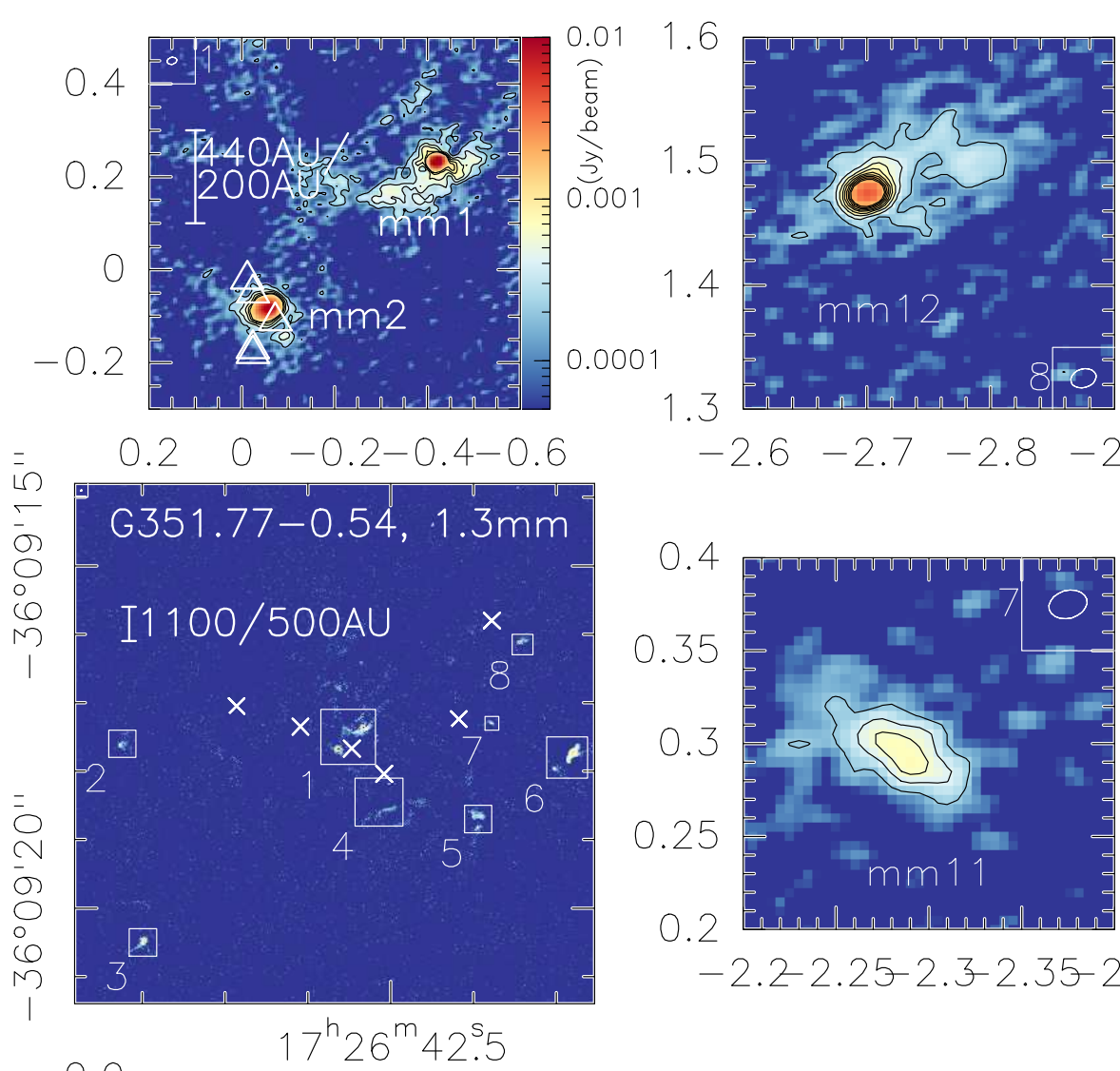

$-2.6-2.7-2.8-2.9$

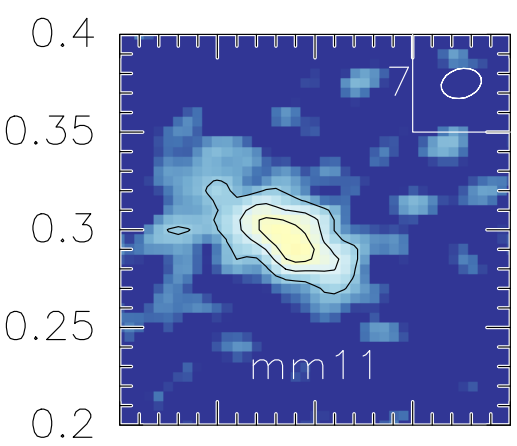

$-2.2-2.25-2.3-2.35-2.4$
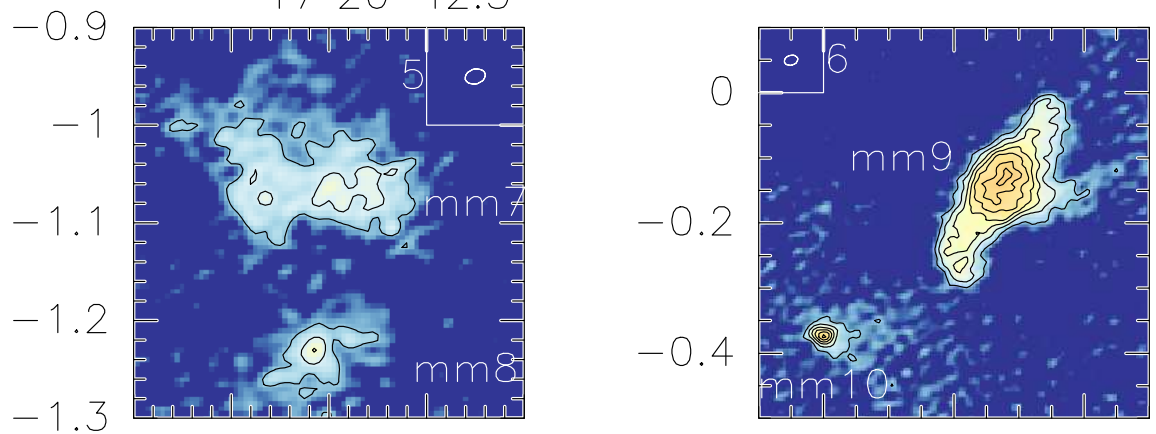

$-3.2-3.4-3.6$

Fig. 2. ALMA $1.3 \mathrm{~mm}$ continuum data toward the high-mass star-forming region G351.77-0.54 at an angular resolution of $0.021^{\prime \prime} \times 0.015^{\prime \prime}$. Central panel: overall structure (the white crosses mark the $\mathrm{cm}$ continuum source positions from Zapata et al. 2008), and the surrounding panels show selected zooms into the areas marked in the central panel. We note the different cutout sizes of the individual zoom windows. The contour levels always start at a $4 \sigma$ level of $0.2 \mathrm{mJy}_{\text {beam }}{ }^{-1}$ and continue in $4 \sigma$ steps. Scale bars assuming 1.0/2.2 kpc distance are shown in the middle and middle top panels. Middle top panel: $\mathrm{CH}_{3} \mathrm{OH}$ maser positions from Walsh et al. (1998) and an intensity scale. The synthesized beam is presented in each panel.

to studies at slightly lower angular resolution (e.g., the NOEMA CORE survey at $\sim 0.4^{\prime \prime}$, Beuther et al. 2018).

What gas temperature would one expect for the outer substructures assuming the heating is dominated by the innermost core $\mathrm{mm} 1$ ? In this case we can do a simple estimate assuming a typical temperature distribution in dust and gas cores of $T \propto r^{-0.4}$ (e.g., van der Tak et al. 2000). Using $1000 \mathrm{~K}$ as the temperature at our resolution element (mean value $0.018^{\prime \prime}$ ), at the approximate spatial separation of the outer core structures of $3.5^{\prime \prime}$ one would get a temperature of $\sim 121 \mathrm{~K}$. Taking into account that (a) the medium is not homogeneous, (b) there are probably several heating sources, and that (c) the $1000 \mathrm{~K}$ at the resolution element is a lower limit because it is an average over the beam size, the measured peak brightness temperatures do not deviate too much from the temperatures one could expect on these scales in such a region if they were heated by an internal source like mm1. It is likely that some of the lower intensity structures already have moderate optical depth at such high spatial resolution as well.

Although we argue that the dust emission has increased optical depth at several positions, we nevertheless would like to get rough estimates of the masses and column densities toward the individual sub-structures. Therefore, we use the typical approach to estimate masses from mm continuum emission assuming optically thin emission (e.g., Hildebrand 1983), but at the same time stress that the derived masses and column densities should be considered as lower limits. Following the equations outlined in Schuller et al. (2009) for dust emission, we are using for the $1.3 \mathrm{~mm}$ dust opacity $\mathrm{K}$ a value of $1.11 \mathrm{~cm}^{2} \mathrm{~g}^{-1}$ from 
Table 1. Millimeter continuum emission parameters.

\begin{tabular}{|c|c|c|c|c|c|c|c|c|c|}
\hline Name & $\begin{array}{r}\text { RA } \\
(\mathrm{J} 2000.0) \\
\end{array}$ & $\begin{array}{r}\text { Dec } \\
(\mathrm{J} 2000.0) \\
\end{array}$ & $\begin{array}{r}S_{\text {peak }} \\
\left(\mathrm{mJy} \mathrm{beam}^{-1}\right) \\
\end{array}$ & $\begin{array}{r}S_{\text {int }} \\
(\mathrm{mJy})\end{array}$ & $\begin{array}{r}T_{\mathrm{b}} \\
(\mathrm{K}) \\
\end{array}$ & $\begin{array}{r}\text { mean } T_{\mathrm{b}} \\
(\mathrm{K}) \\
\end{array}$ & $\begin{array}{r}M @ 1.0 \mathrm{kpc}^{a} \\
\left(M_{\odot}\right) \\
\end{array}$ & $\begin{array}{r}M @ 2.2 \mathrm{kpc}^{a} \\
\left(M_{\odot}\right) \\
\end{array}$ & $\begin{array}{r}N^{b} \\
\left(\times 10^{25} \mathrm{~cm}^{-2}\right)\end{array}$ \\
\hline $\mathrm{mm} 1$ & $17: 26: 42.533$ & $-36: 09: 17.37$ & 15.0 & 47.4 & 1144 & 53 & 0.41 & 2.0 & 3. \\
\hline $\mathrm{mm} 2$ & $17: 26: 42.563$ & $-36: 09: 17.68$ & 13.5 & 42.3 & 1027 & 93 & 0.20 & 1.0 & 3.0 \\
\hline $\mathrm{mm} 3$ & $17: 26: 42.827$ & $-36: 09: 17.63$ & 0.7 & 4.5 & 56 & 24 & 0.10 & 0.5 & 1 . \\
\hline $\mathrm{mm} 4$ & $17: 26: 42.797$ & $-36: 09: 20.50$ & 1.9 & 21.0 & 152 & 44 & 0.22 & 1.1 & 4.5 \\
\hline $\mathrm{mm} 5$ & $17: 26: 42.506$ & $-36: 09: 18.58$ & 0.3 & 3.6 & 29 & 20 & 0.10 & 0.5 & 0.7 \\
\hline $\mathrm{mm} 6$ & $17: 26: 42.503$ & $-36: 09: 18.19$ & 1.4 & 1.4 & 111 & 50 & 0.01 & 0.1 & 3.2 \\
\hline $\mathrm{mm} 7$ & $17: 26: 42.395$ & $-36: 09: 18.67$ & 0.6 & 13.8 & 49 & 26 & 0.27 & 1.3 & 1.4 \\
\hline $\mathrm{mm} 8$ & $17: 26: 42.396$ & $-36: 09: 18.83$ & 0.6 & 3.0 & 52 & 24 & 0.06 & 0.3 & 1. \\
\hline mm9 & $17: 26: 42.280$ & $-36: 09: 17.73$ & 1.4 & 55.3 & 115 & 50 & 0.51 & 2.5 & 3. \\
\hline $\mathrm{mm} 10$ & $17: 26: 42.304$ & $-36: 09: 17.97$ & 1.3 & 3.7 & 101 & 32 & 0.06 & 0.3 & 2.9 \\
\hline mm11 & $17: 26: 42.379$ & $-36: 09: 17.31$ & 0.8 & 2.9 & 62 & 30 & 0.05 & 0.2 & 1.8 \\
\hline $\mathrm{mm} 12$ & $17: 26: 42.345$ & $-36: 09: 16.13$ & 4.0 & 10.5 & 310 & 38 & 0.13 & 0.6 & 9.4 \\
\hline
\end{tabular}

Notes. ${ }^{(a)}$ Masses are calculated using their mean brightness temperature $T_{\mathrm{b}} \cdot{ }^{(b)}$ Column densities are calculated using the peak brightness temperature $T_{\mathrm{b}}$.

Ossenkopf \& Henning (1994) at high densities of $10^{8} \mathrm{~cm}^{-3}$. Furthermore, a gas-to-dust mass ratio of 150 is assumed (Draine 2011). Regarding the temperatures, since we are apparently dealing with optically thick emission, using the derived brightness temperature as an approximate proxy to estimate the actual temperatures seems a reasonable approach. For the column density estimates, we use $1000 \mathrm{~K}$ for the two very bright sources $\mathrm{mm} 1$ and $\mathrm{mm} 2$, and lower temperatures of $100 \mathrm{~K}$ for the other sources (depending on the dust optical depth peak brightness temperatures for some of the weaker sources may be lower limits for the actual gas temperature). Since the substructures are extended and cover a range of brightness temperatures, for the mass estimates, we derive for each core a mean brightness temperature within the $4 \sigma$ contour (Table 1 ).

Table 1 presents the derived masses and column densities, and we stress that these values have to be considered as lower limits because of the high optical depth as well as the large amount of missing flux. Hence, although individual core masses between a fraction of a solar mass and $2 M_{\odot}$ (depending on distance) do not seem very high, since they are lower limits and measured over very small size scales, they already indicate very high densities. For example, the almost spherical source $\mathrm{mm} 2$ has at $1 \mathrm{kpc}$ distance a mass lower limit of $0.2 M_{\odot}$ and a diameter of $\sim 100 \mathrm{au}$. Assuming that the three-dimensional structure of the core resembles a sphere, we can estimate a number density, and we derive high values for the approximate molecular hydrogen densities around $6.8 \times 10^{10} \mathrm{~cm}^{-3}$. The corresponding column densities are on the order of $10^{25} \mathrm{~cm}^{-2}$, corresponding to visual extinction values around $10^{4} \mathrm{mag}$.

\subsection{Emission lines and the dense inner entrained outflow/jet}

As shown in the spectra in Fig. 1, we barely find any line emission signal in these data. On larger scales molecular emission is expected and also found in previous studies of this region (e.g., Beuther et al. 2009, 2017b; Leurini et al. 2011a), however, this emission is filtered out by these long baseline observations. In contrast to this, toward the compact mm peaks the brightness temperatures of the dust are so high that most lines are found only in absorption ${ }^{1}$. However, there are a few lines seen in Fig. 1

1 At the given high spatial resolution and considering our knowledge about the extended continuum and line emission (Beuther et al. 2017b), continuum and line beam filling factors are assumed to be $\sim 1$.
Table 2. Emission line parameters.

\begin{tabular}{|c|c|c|}
\hline $\begin{array}{l}\text { Frequency } \\
(\mathrm{GHz})\end{array}$ & Possible lines & $\begin{array}{r}E_{u} / k \\
(\mathrm{~K}) \\
\end{array}$ \\
\hline 217.10 & $\mathrm{SiO}(5-4) @ 217.10498$ & 31.3 \\
\hline \multirow[t]{2}{*}{217.82} & $\operatorname{HDCO}\left(26_{4,23}-25_{5,20}\right) @ 217.82120$ & 1218 \\
\hline & $\mathrm{CH}_{3} \mathrm{CH}_{2} \mathrm{CN}\left(65_{7,58}-65_{6,59}\right) @ 217.82669$ & 980 \\
\hline \multirow[t]{2}{*}{231.896} & $\mathrm{C}_{2} \mathrm{H}_{3} \mathrm{OCHO}\left(96_{11,85}-95_{12,84}\right) @ 231.89506$ & 1439 \\
\hline & $\mathrm{CH}_{3} \mathrm{CH}_{2} \mathrm{CN}\left(57_{8,50}-58_{5,53}\right) @ 231.90159$ & 783 \\
\hline \multirow[t]{2}{*}{232.029} & $\mathrm{CH}_{2} \mathrm{CHCN}\left(34_{7,28}-35_{6,29}\right) \mathrm{v}_{11}=2 @ 232.02812$ & 1063 \\
\hline & $\mathrm{CH}_{2} \mathrm{CHCN}\left(34_{7,27}-35_{6,30}\right) \mathrm{v}_{11}=2 @ 232.03717$ & 1063 \\
\hline \multirow[t]{3}{*}{232.693} & $\mathrm{H}_{2} \mathrm{O}\left(5_{5,0}-6_{4,3}\right) @ 232.68670^{a}$ & 3462 \\
\hline & $\mathrm{CH}_{2} \mathrm{CHCN}\left(28_{2,27}-28_{1,28}\right) \mathrm{v}_{15}=1 @ 232.68736$ & 683 \\
\hline & $\left(\mathrm{CH}_{3}\right)_{2} \mathrm{CO}\left(43_{16,27}-43_{15,28}\right) \mathrm{AA} @ 232.69487$ & 686 \\
\hline \multirow[t]{2}{*}{234.82} & $\left(\mathrm{CH}_{3}\right)_{2} \mathrm{CO}\left(67_{48,20}-67_{47,21}\right) \mathrm{EE} @ 234.81810$ & 1912 \\
\hline & $\mathrm{HC}_{5} \mathrm{~N}(88-87) \mathrm{v}_{11}=1 @ 234.81890$ & 655 \\
\hline \multirow[t]{2}{*}{235.965} & $\mathrm{HCOOH}\left(47_{6,42}-46_{7,39}\right) @ 235.96447$ & 1337 \\
\hline & $\mathrm{CH}_{2} \mathrm{CHCN}\left(63_{3,60}-64_{2,63}\right) \mathrm{v}_{11}=1 @ 235.96961$ & 1281 \\
\hline
\end{tabular}

Notes. ${ }^{(a)}$ Suggested in Ginsburg et al. (2018).

that are indeed emission lines, even against the strong continuum with a peak brightness temperature above $1100 \mathrm{~K}$. This implies that these spectral line emission features should stem from molecular lines with very high excitation levels $E_{u} / k$, most likely above $1000 \mathrm{~K}$. Identifying these lines is tricky because one finds many possible line candidates in the typical databases (e.g., JPL, Pickett et al. 1998; CDMS, Müller et al. 2001, Splatalogue ${ }^{2}$. Table 2 presents the frequencies of the emission lines as well as possible transition candidates. Except for the $\mathrm{SiO}(5-4)$ line, we only list line candidates with excitation temperatures $E_{u} / k$ above $500 \mathrm{~K}$. We only list two to three possible candidates for each line, but going through the line catalogs, others are possible as well. Since we are mainly interested in the kinematics of the gas in this study, and not so much in the chemistry, the exact line identification is of lower importance here, and in the following, we refer to the lines only by their frequencies.

All compact emission lines can be well imaged and they show mainly emission around $\mathrm{mm} 1$. Since the $217.82 \mathrm{GHz}$ line is in the spectral window with the highest spectral resolution

\footnotetext{
2 https://www.cv.nrao.edu/php/splat/
} 


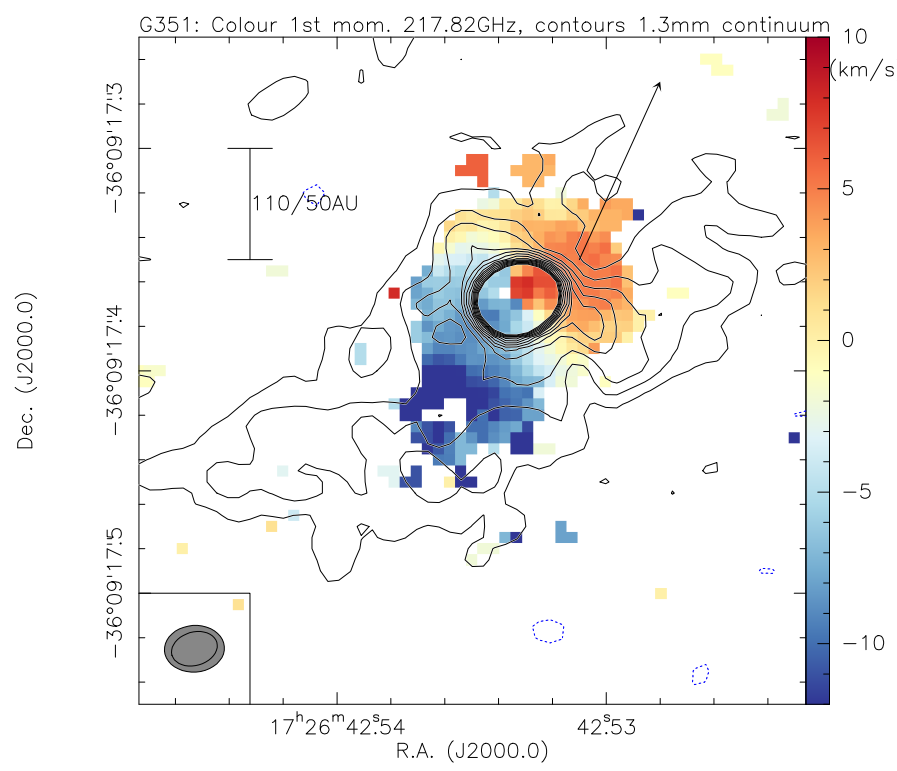

Fig. 3. Color-scale first moment map (intensity-weighted peak velocities) for $\mathrm{mm} 1$ from the line at $217.82 \mathrm{GHz}$. The contours are the $1.3 \mathrm{~mm}$ continuum data in $4 \sigma$ levels of $0.2 \mathrm{mJy}$ beam $^{-1}$ up to a $3 \mathrm{mJy}_{\text {beam }}{ }^{-1}$ level (to avoid too many contours in the center). A scale-bar and the resolution elements of the line (gray) and continuum are shown as well. The arrow outlines the approximate direction of the $\mathrm{CO}(6-5)$ red outflow lobe shown in Fig. 6.

( $244 \mathrm{kHz}$, see Sect. 2), we do most of the analysis with that line. Other line detections toward $\mathrm{mm} 2$ and $\mathrm{mm} 12$ will be discussed in the following Sect. 3.3.

Figure 3 presents a first moment map of the $217.82 \mathrm{GHz}$ line, and one identifies a clear velocity gradient in approximately northwest-southeast direction, similar to the general elongation of the dust continuum emission in this region. For comparison, Fig. 4 shows the corresponding channel map (binned to $2 \mathrm{~km} \mathrm{~s}^{-1}$ ), and one sees that the highest velocity emission is peaked at the northwestern and southeastern sides very close to the continuum peak position whereas the emission in channels around the $v_{\text {lsr }}$ is more evenly distributed around the central continuum peak. In these central channels, the emission is ringlike, however, that is unlikely to reflect the real molecular gas distribution but it is probably due to the high optical depth of the continuum and corresponding self-absorption in that direction. In an alternative representation of the data, the first moment map in Fig. 3 also shows that the highest velocities are close to the center of $\mathrm{mm} 1$ with values of +7.9 and $-9.0 \mathrm{~km} \mathrm{~s}^{-1}$ within a separation of only $\sim 0.01^{\prime \prime}$. Since a moment map measures intensity-weighted peak velocities, peak positions of separate velocity channels can be separated by less than a beam size. A different measure can be the spatial separation of the peak positions in the highest-velocity channels shown in Fig. 4: the spatial peak positions in the channels at -18 and $+14 \mathrm{~km} \mathrm{~s}^{-1}$ are separated by only $0.049^{\prime \prime}$ or $\sim 108 / 49$ au at the two potential distances. Because of the spatially more distributed emission in the intervening channels, peak positions closer to the $v_{\text {lsr }}$ are difficult to measure. Independent of that, the analysis of the first moment map as well as the channel map shows that the highest velocities peak close to the continuum emission peak of $\mathrm{mm} 1$.

The general outflow structures in this region have been discussed in several studies. Leurini et al. (2009, 2011a) suggest three different outflows whereas Klaassen et al. (2015) propose a unified picture where all features may be explained by one outflow structure. The recent ALMA $\mathrm{CO}(6-5)$ data reveal a red-shifted outflow emanating from $\mathrm{mm} 1$ in northwestern direction (Beuther et al. 2017b) which coincides with outflow OF1 from Leurini et al. (2011a). Interestingly, the single outflow suggested by Klaassen et al. (2015) in approximately northeastsouthwest direction has the blue-shifted component toward the north, inverse to what is found in the $\mathrm{CO}(6-5)$ data (Beuther et al. 2017b). Therefore, the interpretation of multiple outflows driven by several sub-sources appears more likely. This also makes sense in the framework of the many $\mathrm{mm}$ and $\mathrm{cm}$ continuum sources identified in this region which are likely to drive several independent outflows.

The fact that one sees the highest-velocity gas in several spectral lines close to the center of source mm1 (Keplerian-like signature), and that the mm continuum emission is elongated in the same direction as the velocity gradient, suggests that these features may be caused by a Keplerian disk. However, several observational features argue against this interpretation. Starting with our knowledge about this region, as shown in our previous ALMA $690 \mathrm{GHz}$ data (Beuther et al. 2017b), the molecular outflow originating from $\mathrm{mm} 1$ is directed in the northwest-southeast direction, similar to the velocity gradient direction in the $217.82 \mathrm{GHz}$ line. Furthermore, the rotational motion visible in the $\mathrm{CH}_{3} \mathrm{CN}\left(37_{k}-36_{k}\right)$ lines at $\sim 680 \mathrm{GHz}$ with almost Keplerian-like motion is oriented perpendicular to the outflow and $217.82 \mathrm{GHz}$ velocity gradient (Fig. 8 in Beuther et al. 2017b). In addition to this, the position-velocity diagram of the $217.82 \mathrm{GHz}$ line along the orientation of the velocity gradient (Fig. 5) shows the high-velocity gas toward the center, but one barely sees any lower velocity gas further out as would be expected for Keplerian motion.

For comparison, we checked whether we can see the outflow in our new $\mathrm{SiO}(5-4)$ data as well. Figure 6 shows the previous $\mathrm{CO}(6-5)$ data (Beuther et al. 2017b) as well as the blueand redshifted $\mathrm{SiO}(5-4)$ emission. Although the outflow is typically more extended, $\mathrm{SiO}$ traces a more compact structure that can also be identified in our data. One sees that the extended redshifted $\mathrm{SiO}(5-4)$ emission traces a collimated jet-like structure emanating from $\mathrm{mm} 1$ toward the northwest that at larger scales spatially coincides with one cavity wall that is also seen in the $\mathrm{CO}(6-5)$ emission. In addition to this, the compact blueand redshifted $\mathrm{SiO}$ emission is also oriented in the northwestsoutheast direction around the mm1 source. This high-velocity $\mathrm{SiO}$ gas shows the same spatial and velocity orientation we also see in the highly excited $217.82 \mathrm{GHz}$ line.

It is interesting to note that in recent ALMA SiO(5-4) observations toward the high-mass disk candidate G17.64 at $\sim 0.2^{\prime \prime}$, the thermal $\mathrm{SiO}$ emission is very compact with a velocity gradient perpendicular to the outflow (Maud et al. 2018). In that case the $\mathrm{SiO}$ emission is interpreted as being emitted from a disk. While thermal $\mathrm{SiO}$ in the past has typically been attributed to shocks from outflows (e.g., Schilke et al. 1997), the G17.64 data indicate also possible different origins of thermal $\mathrm{SiO}$ emission. In that context, we cannot exclude that some of the compact $\mathrm{SiO}$ emission in $\mathrm{G} 351$ toward the center of $\mathrm{mm} 1$ may have a disk-contribution. However, in G351 considering also the more extended $\mathrm{SiO}$ emission, its spatial and velocity-association with the $\mathrm{CO}(6-5)$ emission, and the perpendicular velocity gradient found in $\mathrm{CH}_{3} \mathrm{CN}\left(37_{k}-36_{k}\right)$ (Beuther et al. 2017b), the $\mathrm{SiO}$ emission presented here seems to be dominated by the outflow.

The combination of these various observations indicates that the highly excited line emission we see in our new long baseline ALMA data are likely tracing the innermost jet/outflow driven by $\mathrm{mm} 1$. It is interesting to note that the $1.3 \mathrm{~mm}$ continuum 


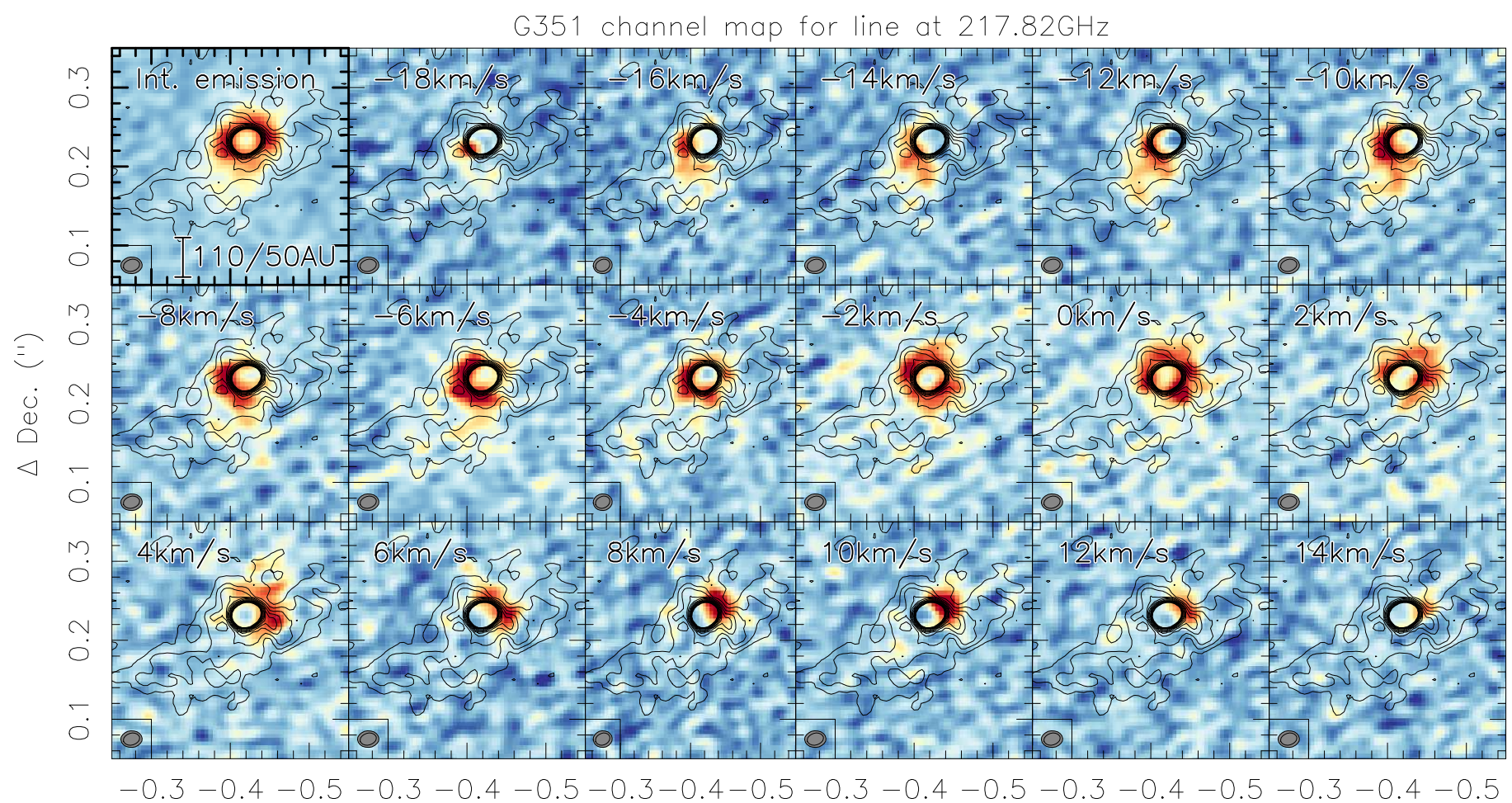

$\triangle$ R.A. ('1)

Fig. 4. Channel map of the $217.82 \mathrm{GHz}$ line for mm1. Top-left panel: integrated emission between -18 and $+14 \mathrm{~km} \mathrm{~s}^{-1}$. The remaining panels show the channels with $2 \mathrm{~km} \mathrm{~s}^{-1}$ width at the velocities marked at the top-left of each panel. The contours are the $1.3 \mathrm{~mm}$ continuum data in $4 \sigma$ level of $0.2 \mathrm{mJy}_{\text {beam }}^{-1}$ up to a $3 \mathrm{mJy}_{\text {beam }}^{-1}$ level (to avoid too many contours in the center). A scale-bar is shown in the top-left panel, and the resolution elements of the line (gray) and continuum are presented in each panel. The $v_{\mathrm{lsr}}$ is at approximately $-3.6 \mathrm{~km} \mathrm{~s}^{-1}$.

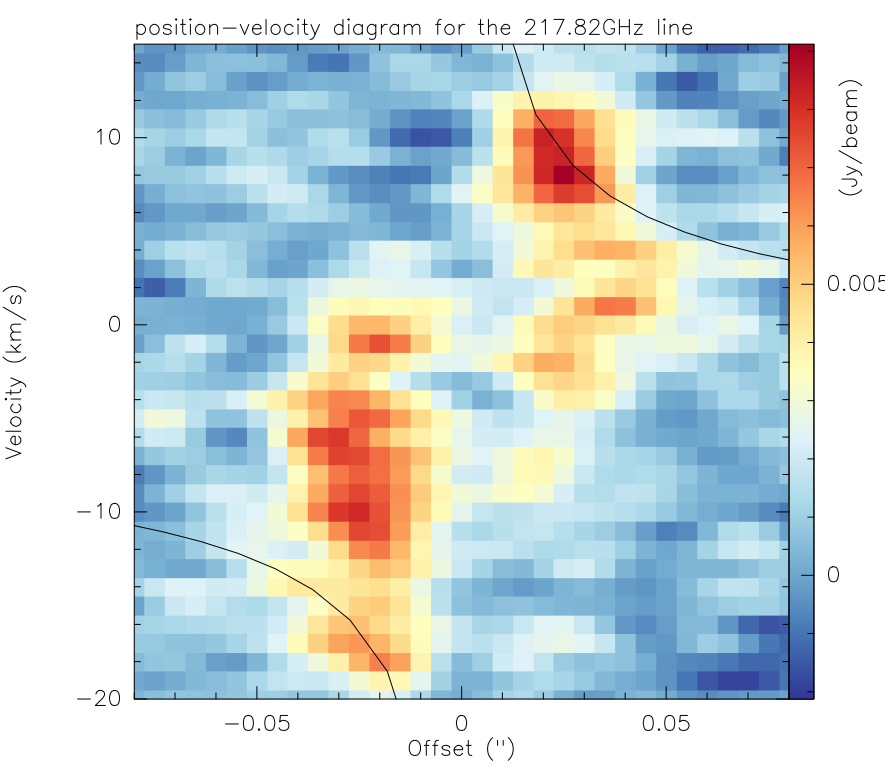

Fig. 5. Color-scale position-velocity diagram for $\mathrm{mm} 1$ for the line at $217.82 \mathrm{GHz}$ along the axis of the strong velocity gradient visible in Fig. 3. The lines correspond to a Keplerian curve around a $10 M_{\odot}$ central object, not representing the data well.

emission is also elongated along the direction of the outflow (Fig. 6). Therefore, this extended $1.3 \mathrm{~mm}$ emission apparently does not stem from an inner disk, but seems rather to be related to the inner jet region of the source as well. Similar outflowrelated dust continuum emission features were found toward the low-mass outflow region L1157 (Gueth et al. 2003). While the southeastern extension of the dust emission is elongated along the outflow direction, the northwestern continuum structure more resembles a cone-like structure often observed in molecular outflows (e.g., Arce \& Sargent 2006). As seen in Fig. 6, these cone-like continuum features toward the northwest form an envelope around the central $\mathrm{SiO}$ jet-like emission, possibly tracing the outflow cavity walls.

To compare the larger scale velocities from $\mathrm{SiO}(5-4)$ with the smaller-scale velocity structure in the $217.82 \mathrm{GHz}$ line, Fig. 7 shows the position-velocity diagram extracted along the axis of the outflow. While the blue-shifted $\mathrm{SiO}$ emission is largely filtered out by our extended baseline configuration, we find $\mathrm{SiO}(5-4)$ emission at almost all redshifted velocities close to the center of mm1. Going further outward in the northwestern direction (positive offsets in Fig. 7), weaker emission is found around velocities of $\sim 10 \mathrm{~km} \mathrm{~s}^{-1}$ but without any strong gradients. None of these data show a "Hubble-law" like velocity distribution, where the velocities would increase with distance from the center (e.g., Downes \& Ray 1999; Arce et al. 2007). Rather the opposite, we find that the highest velocities are close to the center. Implications for the entrainment process are discussed in Sect. 4.2.

\subsection{Emission lines in other cores}

For completeness, we also report detections of emission lines toward a few other sub-sources. We detect line emission from fewer lines toward $\mathrm{mm} 2$ and $\mathrm{mm} 12$, while all other sub-sources remain undetected in emission lines. Figure 8 presents the first moment maps toward $\mathrm{mm} 1, \mathrm{~mm} 2$ and $\mathrm{mm} 12$ in the emission line at $232.693 \mathrm{GHz}$. While for $\mathrm{mm} 1$, the velocity structure 


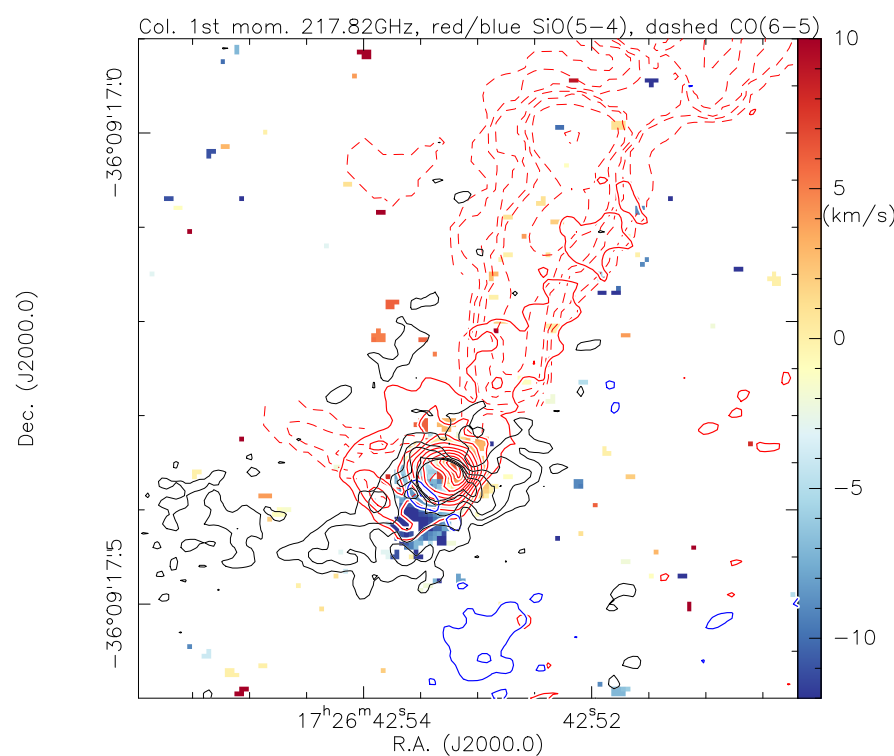

Fig. 6. Color-scale first moment map (intensity-weighted peak velocities) for $\mathrm{mm} 1$ from the line at $217.82 \mathrm{GHz}$. The blue and red solid contours present the blue- and redshifted $\mathrm{SiO}(5-4)$ emission integrated between $[-22,-14]$ and $[0,24] \mathrm{km} \mathrm{s}^{-1}$, respectively. The red dashed contours show the red-shifted $\mathrm{CO}(6-5)$ emission from Beuther et al. (2017b). Blue-shifted $\mathrm{CO}(6-5)$ can not be properly imaged in that dataset, most likely because of missing short spacings. The black contours show the lower level $1.3 \mathrm{~mm}$ continuum data from the $4 \sigma$ levels of 0.2 to $1.0 \mathrm{mJy}^{-1}$ beam $^{-1}$ in $4 \sigma$ steps.

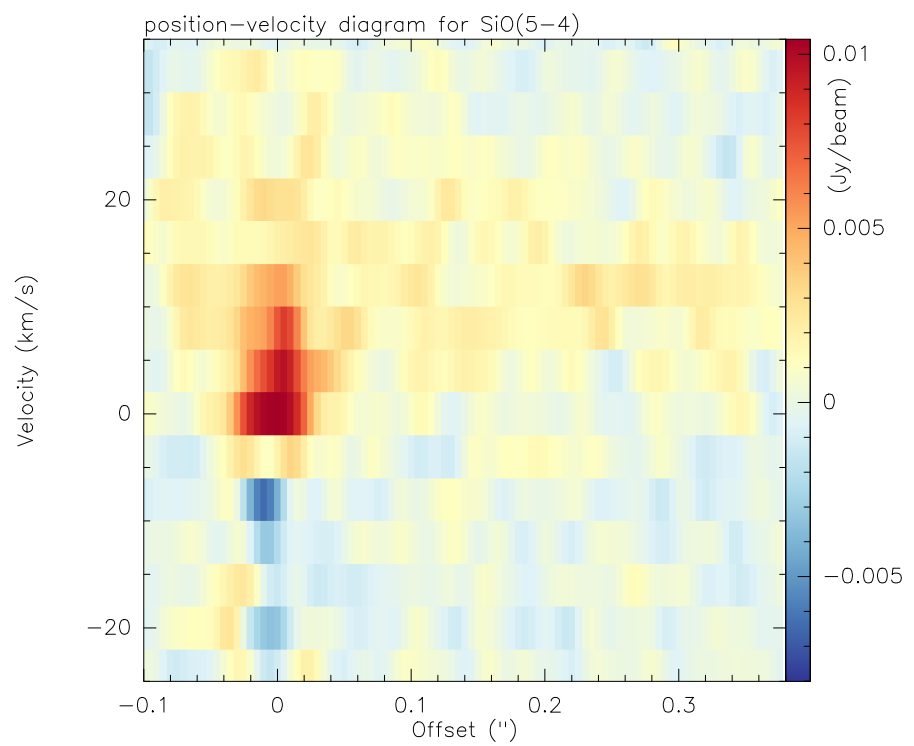

Fig. 7. Color-scale position-velocity diagram from $\mathrm{SiO}(5-4)$ along the outflow axis seen in Fig. 6.

previously found in the $217.82 \mathrm{GHz}$ line is well recovered, it is interesting that the other two sources also depict clear velocity gradients. The one in $\mathrm{mm} 2$ has approximately the same orientation as that in $\mathrm{mm} 1$, however, the blue- and red-shifted sides are swapped. For $\mathrm{mm} 12$, the velocity gradient is in the northsouth direction. Since we do not have any information about molecular outflows from these two sub-sources $\mathrm{mm} 2$ and $\mathrm{mm} 12$ (the $\mathrm{cm}$ continuum emission from Zapata et al. 2008 is not associated with any of these features either), we cannot infer whether these velocity gradients are caused by rotation, outflows or any other process.
For $\mathrm{mm} 2$, an additional feature arises when imaging the line at $231.896 \mathrm{GHz}$ (Fig. 9). While the structure in $\mathrm{mm} 1$ is the same as found in the other lines, toward $\mathrm{mm} 2$ we find emission mainly blue-shifted from the $v_{\mathrm{lsr}}$ approximately -3.6 to $-20 \mathrm{~km} \mathrm{~s}^{-1}$. This emission can either be from the same line, just blue-shifted to high velocities, or it may stem from another spectral line that we cannot differentiate properly. Independent of that, the velocity gradient found in this line is approximately in the northeast-southwest direction, roughly perpendicular to the velocity gradient seen before in the $232.693 \mathrm{GHz}$ line. The perpendicular directions of the velocity gradients in the two lines toward $\mathrm{mm} 2$ indicates that one may trace an outflow/jet whereas the other may trace rotation. Since we do not have any additional information at hand, we refrain from further interpretation of these structures.

\section{Discussion}

\subsection{Fragmentation}

Fragmentation of molecular clouds and star-forming regions is a hierarchical process, and one finds fragmentation from largescale molecular clouds (e.g., Blitz 1993; Kramer et al. 1998; McKee \& Ostriker 2007; Dobbs et al. 2014) to filament fragmentation from giant $100 \mathrm{pc}$ long filaments (Jackson et al. 2010; Goodman et al. 2014; Ragan et al. 2014; Wang et al. 2014, 2015; Zucker et al. 2015; Abreu-Vicente et al. 2016) to those within star-forming regions (e.g., André et al. 2010, 2014; Hacar et al. 2013; Beuther et al. 2015). It is interesting to note that there seems to be a difference in the dominant process driving the fragmentation of filaments and that of star-forming clumps and cores. While filaments can often be described by selfgravitating cylinders (e.g., Jackson et al. 2010; Kainulainen et al. 2013; Beuther et al. 2015), star-forming regions are often welldescribed by thermal and/or turbulent Jeans fragmentation (e.g., Palau et al. 2013, 2014; Wang et al. 2014; Beuther et al. 2018, Svoboda et al., in prep.). While previous studies of fragmentation in (high-mass) star-forming regions typically studied the fragmentation of star-forming regions from scales of several $10^{4}$ au down to scales of a few $1000 \mathrm{au}$, in this study we now zoom into the most central high-mass star-forming core and investigate the fragmentation on scales of hundreds to $1000 \mathrm{au}$.

What would be the thermal Jeans fragmentation properties of a typical high-mass core of several 1000 au size? Using the study of the IRAM large program CORE of 20 high-mass starforming regions, Beuther et al. (2018) find, for regions below $2 \mathrm{kpc}$ distance, typical mean volume number densities between $10^{7}$ and $10^{8} \mathrm{~cm}^{-3}$ averaged over spatial resolution elements of approximately $0.3^{\prime \prime}-0.4^{\prime \prime}$. Assuming mean temperatures of around $50 \mathrm{~K}$ for these regions because the star formation process is already active, we find Jeans lengths between roughly 870 and $2800 \mathrm{au}$, and Jeans masses between 0.1 and $0.4 M_{\odot}$. Although our observed separations and masses are uncertain due to the poorly constrained distance, projection effects as well as missing flux and optical depth uncertainties, the typical observed source separations and masses (Sect. 3.1) are on the order of these Jeans estimates.

One may also ask whether all the identified twelve subsources will form stars. While the compact structures like mm1, $\mathrm{mm} 2, \mathrm{~mm} 4$ or $\mathrm{mm} 12$ seem good candidates for actual forming protostars ( $\mathrm{mm} 1$ is actively driving an outflow, $\mathrm{mm} 2$ and $\mathrm{mm} 12$ are also showing line emission from highly excited lines), other structures like $\mathrm{mm} 5, \mathrm{~mm} 7$ or mm11 appear more diffuse and may simply be local over-densities which could be transient structures 

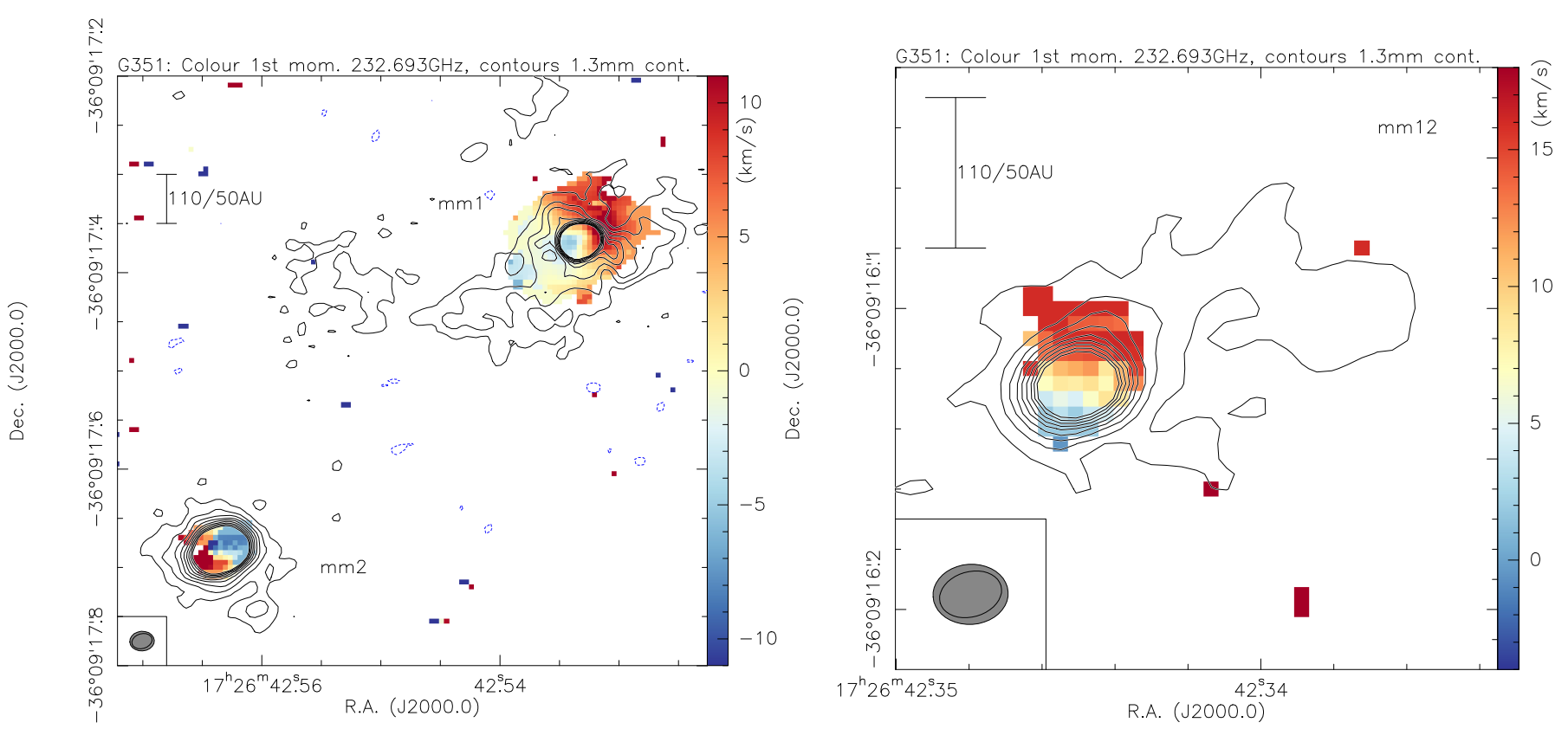

Fig. 8. Color-scale first moment maps (intensity-weighted peak velocities) from the line at $232.693 \mathrm{GHz}$. Left panel: focus on mm1 and mm2. Right panel: $\mathrm{mm} 12$. The contours are the $1.3 \mathrm{~mm}$ continuum data in $4 \sigma$ level of $0.2 \mathrm{mJy} \mathrm{beam}^{-1}$ up to a $3 \mathrm{mJy}$ beam ${ }^{-1}$ level (to avoid too many contours in the center). A scale-bar and the resolution elements of the line (gray) and continuum are shown as well.

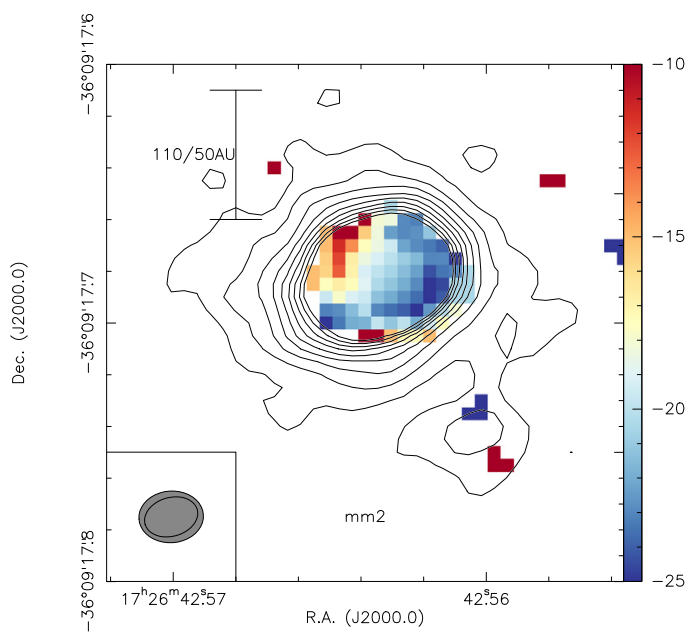

Fig. 9. Color-scale first moment map (intensity-weighted peak velocities) from the line at $231.896 \mathrm{GHz}$ for the source $\mathrm{mm} 2$. The contours are the $1.3 \mathrm{~mm}$ continuum data in $4 \sigma$ level of $0.2 \mathrm{mJy} \mathrm{beam}^{-1}$ up to a $3 \mathrm{mJy}$ beam $^{-1}$ level (to avoid too many contours in the center). A scalebar and the resolution elements of the line (gray) and continuum are shown as well.

and will not form stars within them. In fact, these more diffuse structures may be parts of the otherwise largely filtered out envelope which, if it were infalling, could result in these structures ending up on some protostar later in its evolution.

In this context, the spatial structure of mm9 appears intriguing. Its partly bent and wave-like structure has an appearance similar to gravitationally interacting core structures also observed in recent $3 \mathrm{D}$ radiation-hydrodynamical simulations of high-mass star-forming regions (e.g., Meyer et al. 2018; Ahmadi et al., in prep.). For example, Meyer et al. (2018) model an infalling envelope around a self-gravitating disk that evolves while being irradiated by the time-dependent evolution of the central protostar. Independent of their initial angular velocity distribution, all their models form similarly bent-like structures at scales of several hundred to thousand au from the center. From the observational side, infalling streamer-like structures have recently also been reported in other high-mass star-forming regions (e.g., Maud et al. 2017; Izquierdo et al. 2018; Goddi et al. 2018). In particular, the observations and analysis of the highmass region $\mathrm{W} 33 \mathrm{~A}$ reveals comparably extended structures at $\sim 1000$ au separation from the center that are attributed to spirallike infalling gas streamer (Maud et al. 2017; Izquierdo et al. 2018). If those data were observed with poorer $u v$-coverage, these more extended spiral-like structure may well resemble what we see here in G351.77-0.54. Hence, within the G351.770.54 region, so far, we do not find a larger disk-like structure like for example in AFGL4176 (Johnston et al. 2015) or G17.64 (Maud et al. 2018), but it is more a multisource environment with smaller scale structures like those found in W33A or W51n (e.g., Izquierdo et al. 2018; Goddi et al. 2018).

However, with the given data, such interpretation is for G351.77-0.54 still largely premature and speculative. To investigate this "inter-core" gas in more detail, complementary ALMA observations with shorter baselines are needed. With such data, not only can the more diffuse dust emission be studied, but also the line emission at lower brightness to investigate the kinematics of the connecting gas structures.

\subsection{Turbulent entrainment}

Following the interpretation outlined in Sect. 3.2 that the compact high-energy line emission stems from the innermost jet/outflow driven from $\mathrm{mm} 1$, we can use these data to further analyze the entrainment mechanism. Since large-scale molecular outflows are typically entrained gas and do not directly trace the underlying driving jet, gas entrainment processes are important in the star-formation process. Jet-outflow entrainment processes have been discussed for many years (e.g., Masson \& Chernin 1993; Raga et al. 1993; Stahler 1994; Downes \& Ray 1999; Arce \& Goodman 2002; Cantó et al. 2003; Arce et al. 2007; Frank et al. 2014; Raga 2016). One of the key-diagnostics to study gas entrainment are position-velocity 
diagrams, and one recurring feature in the literature is the socalled Hubble-law of molecular outflows where the observed gas velocities increase with increasing distance from the source (e.g., Raga et al. 1993; Downes \& Ray 1999; Arce et al. 2007). The most discussed outflow entrainment scenarios are turbulent entrainment, jet-bow-shock entrainment as well as wide-angle winds (e.g., Canto \& Raga 1991; Masson \& Chernin 1993; Stahler 1994; Chernin \& Masson 1995; Bence et al. 1996; Cliffe et al. 1996; Li \& Shu 1996; Hatchell et al. 1999; Lee et al. 2001; Arce \& Goodman 2002; Arce et al. 2007; Frank et al. 2014). A summary of these processes and the corresponding diagnostics, in particular the position-velocity diagrams for the various processes can be found in Arce et al. (2007). While the promptentrainment jet-bow-shock models and the wide-angle winds both predict velocity structures where the velocity increases with distance to the source, the more steady-state turbulent entrainment predicts rather the opposite with high velocities close to the source (Fig. 2 in Arce et al. 2007). Interestingly, many outflow studies on larger spatial scales seem to prefer the jet-bow-shock and wide-angle models (e.g., Yu et al. 1999; Lee et al. 2002; Arce \& Goodman 2002; Arce et al. 2007; Ray et al. 2007; Tafalla et al. 2017).

However, although the central peak position around $\mathrm{mm} 1$ suffers from absorption (e.g., Figs. 1 and 4), our new data of the hot, likely entrained gas around $\mathrm{mml}$ do not show any Hubble-like velocity structure with increasing velocity away from the center, but we clearly find high-velocity gas very close to the center of the source (Sect. 3.2, and Figs. 3 and 4). Furthermore, also the position-velocity diagram of $\mathrm{SiO}(5-4)$ along the outflow axis exhibits the highest velocities close to the center and then almost constant velocities with increasing distance from the center (Fig. 7). Hence, also out to distances of several hundred au from the center of mm1, the new ALMA long baseline data do not show Hubble-like velocity-structure. We stress that to our knowledge barely any high-mass jet study in thermal line emission so far has ever resolved the scales below 50 au from the driving protostar as we do here (a notable exception is source I in Orion, Ginsburg et al. 2018). Hence, we reach new territory with the supreme capabilities of ALMA. Compared to the basic predictions of the prompt bow-shock entrainment models at the heads of the jets, and the steady-state turbulent entrainment models via Kelvin-Helmholtz instabilities along the jets (e.g., Bence et al. 1996; Arce \& Goodman 2002; Arce et al. 2007), on these small scales, the position-velocity diagnostics are more consistent with turbulent entrainment at the jet-outflow interface. From a physical point of view, both processes have to exist, steady-state turbulent entrainment via Kelvin-Helmholtz instabilities along the jet-envelope interface as well as prompt entrainment at the head of jets via bow-shocks. Therefore, the question is not that much about the existence of the different processes but rather which of them dominates in one or the other source and at which spatial scales. Hence, the results for G351.77-0.54 do not necessarily contradict previous observational results that found the Hubble-like velocity structure on larger spatial scales. It is rather possible that on the smallest scales, as observed here, turbulent entrainment may be more important whereas on larger scales bow-shock entrainment could become the dominant process. This issue can again only be directly tackled by observations that connect all spatial scales, from those observed in this study of G351.77 to the larger scales of the more typically observed outflows.

An additionally interesting feature with respect to the inner jet/outflow and accretion disk is the extension of the $1.3 \mathrm{~mm}$ continuum emission along the axis of the jet/outflow. While elongations of $1.3 \mathrm{~mm}$ continuum emission are typically attributed to the dense cores and potential accretion disks, this seems less likely for the case of G351.77-0.54 here. As outlined in Sect. 3.2 and Fig. 3, while toward the southeast of mm1 the $1.3 \mathrm{~mm}$ continuum emission is elongated along the outflow axis, toward the northwest, the mm emission rather resembles a cone-like structure almost enveloping the central $\mathrm{SiO}(5-4)$ jet and $\mathrm{CO}(6-5)$ outflow emission. While we do not have a reasonable explanation for this asymmetry of the $1.3 \mathrm{~mm}$ emission, both features appear qualitatively consistent with entrainment of the ambient dust and gas. It may be that for the blue wing, we see into the outflow lobe and trace the more compact structures, whereas the red wing could cover the central jet better and one would see more of the limb-brightened edges. As discussed in Sect. 4.1, typical mean densities in these dense cores are $10^{7}-10^{8} \mathrm{~cm}^{-3}$ (e.g., Beuther et al. 2018), and close to the peak positions even higher (Sect. 3.1). At such high densities, gas and dust are well coupled, and if the gas is entrained by the underlying jet, it should be no surprise that similar spatial structures are also observed in the dust continuum emission.

\section{Conclusions}

Resolving for one of the first times a high-mass star-forming region at sub-50 au spatial scales ( 18 or 40 au resolution depending on the distance of 1.0 or $2.2 \mathrm{kpc}$ ) reveals various highly interesting outcomes. In the dust continuum emission, we resolve twelve distinct sub-sources within a region of roughly $6^{\prime \prime} \times 6^{\prime \prime}$ or $6000 / 13200 \mathrm{au}$ at $1.0 / 2.2 \mathrm{kpc}$ distance. Projected separations between sub-sources range from more than $1000 \mathrm{au}$ to several 100 au but can be even smaller below 100 au. Since we filter out the large-scale emission, very extended structures are not visible in the data, but the largest features we can identify are between approximately 200 and 600 au in size, similar to the size-scales predicted by theoretical simulations for disks around embedded high-mass protostars. Brightness temperatures toward the main continuum peak positions are in excess of $1000 \mathrm{~K}$, indicating high optical depth toward these positions. This high optical depth as well as the missing flux only allows us to derive lower limits for the masses and column densities of the sub-sources. In the hierarchical picture of fragmentation from large-scale clouds to small-scale cores, we find that the fragmentation properties of this high-mass core with a size of several 1000 au is consistent with thermal Jeans fragmentation.

The high continuum optical depth is also confirmed by the fact that most spectral lines toward the dust peak positions are only seen in absorption. However, we find a few lines in emission toward the main source $\mathrm{mm} 1$ as well as two other sources $\mathrm{mm} 2$ and $\mathrm{mm} 12$. Because of the high dust continuum brightness temperatures, these emission lines have to stem from transitions with very high excitation levels $E_{u} / k$ on the order of $1000 \mathrm{~K}$. The emission toward $\mathrm{mm} 1$ shows a velocity gradient with the highest velocities found toward the core center. While this highexcitation line emission by itself may be indicative of a Keplerian disk-like structure, additional information let us favor a different interpretation. In particular, the elongation of this velocity gradient is exactly along the direction of the molecular outflow observed in $\mathrm{SiO}(5-4)$ and $\mathrm{CO}(6-5)$ and perpendicular to the rotational velocity gradient previously identified in $690 \mathrm{GHz}$ $\mathrm{CH}_{3} \mathrm{CN}\left(37_{k}-36_{k}\right)$ data (Beuther et al. 2017b). Hence, this gas emission does likely not arise from an inner accretion disk but is more consistent with being caused by the central jet/outflow region. The high-velocity gas close to the center is consistent with steady-state turbulent entrainment of the molecular gas 
from the underlying jet via Kelvin-Helmholtz instabilities. So far, in G351.77-0.54 we do not identify a larger scale disk but the region is composed of a smaller scale multisource environment.

Since this study only covers the smallest spatial scales without any baselines below $55 \mathrm{~m}$, we are not seeing any extended emission. Such extended emission would be important to connect the scales from thousands and more au down to sub-50 au scales observed here. How does the dense compact structure connect to the more extended gas? How are the kinematic connections from large to small spatial scales? These questions can only be addressed by data with a more complete $u v$-coverage that would trace all spatial scales and would also be sensitive to lower brightness emission from dust and gas. These investigations will be conducted in future studies.

Acknowledgements. The proposal and design of this study was devised in close collaboration with Malcolm Walmsley who unfortunately passed away in 2017 We like to express our deep gratitude to Malcolm for many years of close collaboration, which was always a delight and very inspiring! We also like to thank a lot an anonymous referee for detailed comments improving the paper. This paper makes use of the following ALMA data: ADS/JAO.ALMA\#2015.1.00496.S ALMA is a partnership of ESO (representing its member states), NSF (USA) and NINS (Japan), together with NRC (Canada) and NSC and ASIAA (Taiwan) and KASI (Republic of Korea), in cooperation with the Republic of Chile. The Joint ALMA Observatory is operated by ESO, AUI/NRAO and NAOJ. H.B., A.A., and J.C.M. acknowledge support from the European Research Council under the Horizon 2020 Framework Program via the ERC Consolidator Grant CSF-648505. R.K. acknowledges financial support from the Emmy Noether Research Program, funded by the German Research Foundation (DFG) under grant number KU 2849/3-1.

\section{References}

Abreu-Vicente, J., Ragan, S., Kainulainen, J., et al. 2016, A\&A, 590, A131 ALMA Partnership (Brogan, C. L., et al.) 2015, ApJ, 808, L3

André, P., Men'shchikov, A., Bontemps, S., et al. 2010, A\&A, 518, L102

André, P., Di Francesco, J., Ward-Thompson, D., et al. 2014, in Protostars and Planets VI, eds. H. Beuther, R. Klessen, C. Dullemond, \& T. Henning (Tucson, AZ: University of Arizona Press), 27

Arce, H. G., \& Goodman, A. A. 2002, ApJ, 575, 928

Arce, H. G., \& Sargent, A. I. 2006, ApJ, 646, 1070

Arce, H. G., Shepherd, D., Gueth, F., et al. 2007, in Protostars and Planets V, eds B. Reipurth, D. Jewitt, \& K. Keil (Tucson, AZ: University of Arizona Press), 245

Beltrán, M. T., \& de Wit W. J. 2016, A\&ARv, 24, 6

Bence, S. J., Richer, J. S., \& Padman, R. 1996, MNRAS, 279, 866

Beuther, H., Zhang, Q., Bergin, E. A., et al. 2007, A\&A, 468, 1045

Beuther, H., Walsh, A. J., \& Longmore, S. N. 2009, ApJS, 184, 366

Beuther, H., Linz, H., \& Henning, T. 2013, A\&A, 558, A81

Beuther, H., Ragan, S. E., Johnston, K., et al. 2015, A\&A, 584, A67

Beuther, H., Linz, H., Henning, T., Feng, S., \& Teague, R. 2017a, A\&A, 605, A61

Beuther, H., Walsh, A. J., Johnston, K. G., et al. 2017b, A\&A, 603, A10

Beuther, H., Mottram, J. C., Ahmadi, A., et al. 2018, A\&A, 617, A100

Blitz, L. 1993, in Protostars and Planets III (Tucson, AZ: University of Arizona Press), 125

Bontemps, S., Motte, F., Csengeri, T., \& Schneider, N. 2010, A\&A, 524, A18

Canto, J., \& Raga, A. C. 1991, ApJ, 372, 646

Cantó, J., Raga, A. C., \& Riera, A. 2003, Rev. Mex. Astron. Astrofis., 39, 207

Cesaroni, R., Sánchez-Monge, Á., Beltrán, M. T., et al. 2017, A\&A, 602, A59

Chernin, L. M., \& Masson, C. R. 1995, ApJ, 455, 182

Cliffe, J. A., Frank, A., \& Jones, T. W. 1996, MNRAS, 282, 1114

Csengeri, T., Bontemps, S., Wyrowski, F., et al. 2017, A\&A, 600, L10

Dobbs, C. L., Krumholz, M. R., Ballesteros-Paredes, J., et al. 2014, Protostars and Planets VI (Tucson, AZ: University of Arizona Press), 3
Downes, T. P., \& Ray, T. P. 1999, A\&A, 345, 977

Draine, B. T. 2011, Physics of the Interstellar and Intergalactic Medium, Princeton Series in Astrophysics (Princeton: Princeton University Press)

Frank, A., Ray, T. P., Cabrit, S., et al. 2014, Protostars and Planets VI (Tucson, AZ: University Press of Arizona), 451

Ginsburg, A., Bally, J., Goddi, C., Plambeck, R., \& Wright, M. 2018, ApJ, 860, 119

Goddi, C., Ginsburg, A., Maud, L., Zhang, Q., \& Zapata, L. 2018, ArXiv e-prints [arXiv:1805.05364]

Goodman, A. A., Alves, J., Beaumont, C. N., et al. 2014, ApJ, 797, 53

Gueth, F., Bachiller, R., \& Tafalla, M. 2003, A\&A, 401, L5

Hacar, A., Tafalla, M., Kauffmann, J., \& Kovács, A. 2013, A\&A, 554, A55

Hatchell, J., Fuller, G. A., \& Ladd, E. F. 1999, A\&A, 346, 278

Hildebrand, R. H. 1983, QJRAS, 24, 267

Izquierdo, A. F., Galván-Madrid, R., Maud, L. T., et al. 2018, MNRAS, 478, 2505

Jackson, J. M., Finn, S. C., Chambers, E. T., Rathborne, J. M., \& Simon, R. 2010, ApJ, 719, L185

Johnston, K. G., Robitaille, T. P., Beuther, H., et al. 2015, ApJ, 813, L19

Kainulainen, J., Ragan, S. E., Henning, T., \& Stutz, A. 2013, A\&A, 557, A120

Klaassen, P. D., Johnston, K. G., Leurini, S., \& Zapata, L. A. 2015, A\&A, 575, A54

Kramer, C., Stutzki, J., Rohrig, R., \& Corneliussen, U. 1998, A\&A, 329, 249

Lee, C., Stone, J. M., Ostriker, E. C., \& Mundy, L. G. 2001, ApJ, 557, 429

Lee, C., Mundy, L. G., Stone, J. M., \& Ostriker, E. C. 2002, ApJ, 576, 294

Leurini, S., Codella, C., Zapata, L. A., et al. 2009, A\&A, 507, 1443

Leurini, S., Codella, C., Zapata, L., et al. 2011a, A\&A, 530, A12

Leurini, S., Pillai, T., Stanke, T., et al. 2011b, A\&A, 533, A85

Leurini, S., Codella, C., Gusdorf, A., et al. 2013, A\&A, 554, A35

Li, Z.-Y., \& Shu, F. H. 1996, ApJ, 468, 261

Müller, H. S. P., Thorwirth, S., Roth, D. A., \& Winnewisser, G. 2001, A\&A, 370, L49

Masson, C. R., \& Chernin, L. M. 1993, ApJ, 414, 230

Maud, L. T., Hoare, M. G., Galván-Madrid, R., et al. 2017, MNRAS, 467, L120

Maud, L. T., Cesaroni, R., Kumar, M. S. N., et al. 2018, A\&A, 620, A31

McKee, C. F., \& Ostriker, E. C. 2007, ARA\&A, 45, 565

McMullin, J. P., Waters, B., Schiebel, D., Young, W., \& Golap, K. 2007, in Astronomical Data Analysis Software and Systems XVI, eds. R. A. Shaw, F. Hill, \& D. J. Bell, ASP Conf. Ser., 376, 127

Meyer, D. M.-A., Kuiper, R., Kley, W., Johnston, K. G., \& Vorobyov, E. 2018, MNRAS, 473, 3615

Moscadelli, L., \& Goddi, C. 2014, A\&A, 566, A150

Norris, R. P., Whiteoak, J. B., Caswell, J. L., Wieringa, M. H., \& Gough, R. G. 1993, ApJ, 412, 222

Ossenkopf, V., \& Henning, T. 1994, A\&A, 291, 943

Palau, A., Fuente, A., Girart, J. M., et al. 2013, ApJ, 762, 120

Palau, A., Estalella, R., Girart, J. M., et al. 2014, ApJ, 785, 42

Pickett, H. M., Poynter, R. L., Cohen, E. A., et al. 1998, J. Quant. Spectr. Rad. Transf., 60, 883

Preibisch, T., Balega, Y., Hofmann, K.-H., Weigelt, G., \& Zinnecker, H. 1999, New Astron., 4, 531

Raga, A. C. 2016, Rev. Mex. Astron. Astrofis., 52, 311

Raga, A. C., Canto, J., Calvet, N., Rodriguez, L. F., \& Torrelles, J. M. 1993, A\&A, 276, 539

Ragan, S. E., Henning, T., Tackenberg, J., et al. 2014, A\&A, 568, A73

Ray, T., Dougados, C., Bacciotti, F., Eislöffel, J., \& Chrysostomou, A. 2007, Protostars and Planets V (Tucson, AZ: University Press of Arizona), 231

Schilke, P., Walmsley, C. M., Pineau des Forets, G., \& Flower, D. R. 1997, A\&A, 321, 293

Schuller, F., Menten, K. M., Contreras, Y., et al. 2009, A\&A, 504, 415

Stahler, S. W. 1994, ApJ, 422, 616

Tafalla, M., Su, Y.-N., Shang, H., et al. 2017, A\&A, 597, A119

Tan, J. C., Beltrán, M. T., Caselli, P., et al. 2014, Protostars and Planets VI (Tucson, AZ: University Press of Arizona), 149

van der Tak, F. F. S., van Dishoeck, E. F., \& Caselli, P. 2000, A\&A, 361, 327

Walsh, A. J., Burton, M. G., Hyland, A. R., \& Robinson, G. 1998, MNRAS, 301, 640

Wang, K., Zhang, Q., Testi, L., et al. 2014, MNRAS, 439, 3275

Wang, K., Testi, L., Ginsburg, A., et al. 2015, MNRAS, 450, 4043

Yu, K. C., Billawala, Y., \& Bally, J. 1999, AJ, 118, 2940

Zapata, L. A., Leurini, S., Menten, K. M., et al. 2008, AJ, 136, 1455

Zucker, C., Battersby, C., \& Goodman, A. 2015, ApJ, 815, 23 